Revista de Indias, 1999, vol. LIX, núm. 216

\title{
INDIANOS PRECURSORES DE LA FILANTROPÍA DOCENTE EN GALICIA (1607-1699)
}

\author{
Ó final a terra énos sempre fiel; \\ énos fiel como nai fecunda e como tumba
}

Xoan Rof Carballo

POR

\author{
VICENTE PEÑA SAAVEDRA
}

Universidad de Santiago de Compostela

\begin{abstract}
En este artículo se realiza un primer recuento y análisis de las acciones filantrópicas emprendidas en el área educativa por los indianos gallegos durante el siglo XVII, preludio de un proceso de larga duración que se prolongará hasta nuestros días. Las fuentes utilizadas son de carácter documental y bibliográfico. El contenido del trabajo se centra en el perfil personal y profesional de los donantes, su asentamiento geográfico, la cadencia de las contribuciones efectuadas, su dimensión financiera, los destinatarios y destinos concretos que les confieren, las realizaciones a que dan lugar y las motivaciones subyacentes a sus remesas. El balance provisional del recuento arroja un total de doce benefactores y un caudal de iniciativas relativamente apreciable y plural.
\end{abstract}

Es un hecho ya historiográficamente constatado que el proceso de institucionalización y difusión de la enseñanza primaria pública y privada en Galicia durante el último cuarto del siglo XIX y, más aún, en el primer tercio del XX, se vio favorecido por los flujos de remesas monetarias que los emigrantes transoceánicos transfirieron desde América a sus respectivas localidades de procedencia ${ }^{1}$.

1 Así lo hemos demostrado en nuestra obra Éxodo, organización comunitaria e intervención escolar. La impronta educativa de la emigración transoceánica en Galicia, 2 vols., Santiago de Compostela, Secretaría Xeral para as Relacións coas Comunidades Galegas da Xunta de Galicia, 1991. Véanse también: Antón COSTA RICO, «La emigración gallega y su acción cultural-educativa en sus lugares de origen», Indianos. Monografías de Los Cuadernos del Norte, $\mathrm{n}^{\circ} 2$, Oviedo, 1984, pp. 35-44; Escolas e mestres. A educación en Galicia: da Restauración á Segunda República, Santiago de Compostela, Servicio Central de Publicacións da Consellería da Presidencia e Admi-

R. I., $1999, \mathrm{n}^{\circ} 216$ 
Pero esta acción de apoyo, estímulo y refuerzo, de índole particular, no quedó acotada a esa secuencia cronológica de entresiglos, como en algunas ocasiones se ha dado a entender, ni sectorialmente restringida al nivel educativo elemental, como se podría sospechar. Tampoco, por supuesto, se limitó al ámbito territorial gallego, conforme acreditan los testimonios de los coetáneos ${ }^{2}$ y corroboran las investigaciones recientes ${ }^{3}$. Hoy sabemos que la intervención en el área educativa de los transterrados a la otra orilla del Atlántico constituye un fenómeno estruc-

nistración Pública, 1989, e «As Sociedades dos 'americanos' e a educación en Galicia. Revista da Comisión Galega do Quinto Centenario, ${ }^{\circ}$ 5, Santiago de Compostela, 1989, pp. 89-139; Consuelo NARANJo OROVIO, Del campo a la bodega: recuerdos de gallegos en Cuba (siglo XX), Sada, Ediciós do Castro, 1988, especialmente pp. 139-158.

2 El más amplio -aunque incompleto- y sistemático está condensado en la obra hoy ya clásica de Benito CASTRILLO SAGREDO, El aporte de los indianos a la instrucción pública, a la beneficencia y al progreso en general de España y su historia hecha en La Prensa de Buenos Aires, Oviedo, Tipografía Región, 1926. Además de este trabajo hay que citar las numerosas referencias al tema que aparecen en las publicaciones periódicas de la época, de uno y otro lado del mar.

3 Véanse, para ASTURIAS: Jorge URÍA, «Los indianos y la instrucción pública en Asturias», Indianos. Monografías de Los Cuadernos del Norte, $\mathrm{n}^{\circ} 2$, Oviedo, 1984, pp. 102-119; Aida TERRóN BAÑUElos y Ángel MATO DíAZ (Coords.), Un modelo escolar integrador y reformista: la fundación escuelas Selgas, Oviedo, KRK ediciones, 1992; Ángel MATo DíAZ, La escuela primaria en Asturias (1923-1937), Oviedo, Ministerio de Educación y Ciencia. Dirección Provincial de Asturias, pp. 102-115; María Cruz MORALES SARO, «Las fundaciones de los indianos en Asturias», en Nicolás SÁNCHEZ ALBORNOZ (Comp.), Españoles hacia América. La emigración en masa, 18801930, Madrid, Alianza Editorial, 1988, pp. 66-79; Juaco LÓPEZ ÁlVAREZ, «Emigración y localismo. Sociedades asturianas en La Habana», Astura, nº 9, 1993, pp. 53-59; Juaco LóPEZ ÁlLVAREZ, «Emigración y localismo: el Club Allandés de La Habana», en Pedro GómEz GómEZ, De Asturias a América. Cuba (1850-1930). La comunidad asturiana de Cuba, Principado de Asturias, 1996, pp. 311-333. Para el PAÍS VASCO: José Manuel AZCONA, Inés GARCía ALBI y Fernando MuRU, Historia de la emigración vasca a Argentina en el $s . X X$, Vitoria, Servicio Central de Publicaciones del Gobierno Vasco, 1992, en particular pp. 322-324; Juan Manuel GonZÁlez CEMBELlín, América en el País Vasco. Inventario de elementos patrimoniales de origen americano en la Comunidad Autónoma Vasca, Vitoria, Servicio de Publiciones del Gobierno Vasco, 1993. Para LA RIOJA: Miguel ZAPATER CORNEJO, Contribución de los emigrantes a la educación en la Rioja. Las fundaciones escolares riojanas decimonónicas, Logroño, Gobierno de La Rioja-Instituto de Estudios Riojanos, 1991; Ernesto ReINARES MARTíneZ y Miguel ZAPATER CoRNeJo, La escuela de San Román de Cameros y sus fundadores comerciantes riojanos en el Méjico colonial, Logroño, Asociación «Amigos de San Román de Cameros», 1987. Para PORTUGAL: Jorge FernANDES AlveS, «Percursos de um brasileiro do Porto-O Conde de Ferreira», Revista da Faculdade de Letras - Historia, II Série, vol. IX, Porto, 1992, pp. 199-213, y Os brasileiros. Emigração e retorno no Porto oitocentista, Porto, Gráficos Reunidos, Lda., 1994, especialmente pp. 315-319 y 326-331. Desde una perspectiva más panorámica Moisés LLORDÉN MIÑAMBRES, «Las asociaciones españolas de emigrantes», en María Cruz Morales SARo y Moisés LloRdÉN MiÑAMBRES (Eds.), Arte, cultura y sociedad en la emigración española a América, Oviedo, Servicio de Publicaciones de la Universidad de Oviedo, 1992, pp. 9-55, e «O asociacionismo dos emigrantes españois en América (18401930). Unha explicación histórica do feito». Estudios Migratorios, $n^{\circ} 2$, Santiago de Compostela, decembro 1996, pp. 39-84.

\section{R. I., $1999, \mathrm{n}^{\circ} 216$}


tural, de más de cuatrocientos años de duración en algunas zonas, que adoptó múltiples variantes y registró diversas ramificaciones en su dilatada singladura ${ }^{4}$.

Por ello, una vez establecidas las grandes coordenadas del tema en su conjunto - al menos en lo que atañe al caso gallego_-, se hace indispensable una segmentación del mismo en unidades de análisis más reducidas y practicables que le permitan al investigador profundizar en el conocimiento de sus peculiaridades, en una doble vertiente: espacial y temporal. Guiados por este criterio, en las páginas que siguen procederemos a realizar una primera aproximación panorámica al estudio de las fundaciones y remesas escolares debidas a la iniciativa de los indianos gallegos en el siglo XVII, período que corresponde a su etapa auroral.

\section{PRIMEROS DONANTES, PRIMERAS DONACIONES}

Todavía no estamos en condiciones de ofrecer un censo exhaustivo y pormenorizado de las fundaciones docentes que durante el siglo XVII - primero del que poseemos referencias documentales - crearon los indianos gallegos en sus pagos de origen, ni de las aportaciones monetarias o de otro tipo que canalizaron hacia el sector educativo. Pero, a pesar de esta limitación, no cabe duda de que el caudal de conocimientos disponibles en torno a la materia que nos ocupa se ha ido incrementando sensiblemente en el transcurso de los últimos años.

Los datos fundamentales que hasta el momento logramos reunir acerca de las primeras contribuciones y de sus donantes los hemos compendiado y clasificado en el cuadro 1, que insertamos al final del presente trabajo. De su lectura se desprende que el número de benefactores identificados como indianos durante toda la centuria asciende a doce, si bien dos de ellos figuran en calidad de cofundadores (Pedro Mondragón y Alonso de Santana), con lo cual la relación de agentes puede cifrarse en once. Téngase en cuenta, por otra parte, que uno de los dos mecenas citados (Pedro Mondragón) no era natural ni siquiera oriundo de Galicia, sino de ascendencia guipuzcoana, y aunque el importe de su legado sirvió de

4 Del fenómeno, en su dimensión gallega, nos hemos ocupado entre otros trabajos en: «As remesas escolares, benéficas e sociais dos emigrados», Galicia e América. Cinco séculos de historia, Santiago de Compostela, Consellería de Relacións Institucionais e Portavoz do GobernoConsello da Cultura Galega, 1992, pp. 207-212; «Das fundacións docentes dos indianos ás escolas de americanos: catro séculos de intervención escolar dos emigrantes galegos», en $I^{\text {os }}$ Encontros Galicia-América, Santiago de Compostela, Cihuga, 1992, pp. 53-79; «As remesas escolares dos emigrantes galegos: o caso cubano», en José Martí en Galicia, Vigo, Asociación de Amistade Galego-Cubana «Francisco Villamil», 1993, pp. 53-61; «Cuatro siglos de intervención escolar de los gallegos de América en la Galicia escindida», Historia de la Educación. Revista interuniversitaria, $\mathrm{n}^{\text {os }} 14-15$, Salamanca, 1995-96, pp. 301-332; «As Sociedades galegas de instrucción: proxecto educativo e realizacións escolares», Estudios Migratorios, $\mathrm{n}^{\circ} 1$, Santiago de Compostela, diciembre 1995, pp. 8-83. 
base financiera o capital fundacional de una obra filantrópica que andando el tiempo se materializó en territorio gallego, la responsabilidad de formalizar y de hacer realmente efectiva la donación, destinándola a Ourense, recayó de manera exclusiva en Alonso de Santana —natural de Vilar de Sandiás, localidad de la comarca de A Limia - quien en definitiva ejerció el papel de «auténtico fundador» ${ }^{5}$.

Aparentemente el índice de donantes podría calificarse de muy modesto en términos absolutos. Pero esta apreciación no sería ajustada en términos relativos, a tenor de la débil presencia demográfica de los gallegos en Indias durante los siglos XVI, XVII y aun en la primera mitad o tal vez en los tres primeros cuartos del XVIII ${ }^{6}$, en rotundo contraste con su masiva afluencia ulterior hacia aquellas tierras ${ }^{7}$. Cabe la posibilidad además de que el elenco real de los benefactores indianos sea algo superior al cómputo anotado, puesto que poseemos referencias fragmentarias de algunos titulares de fundaciones o remitentes de donativos, co-

5 Evaristo RIVERA VÁzQUEZ, Galicia y los jesuitas. Sus colegios y enseñanza en los siglos XVI al XVIII, A Coruña, Fundación «Pedro Barrié de la Maza Conde de Fenosa», 1989, pp. 330335, y «Los jesuitas de Galicia y su presencia en la América española», en Galicia y la evangelización de América. Santiago de Compostela, Secretaría Xeral para as Relacións coas Comunidades Galegas da Xunta de Galicia, 1991, pp. 206-207; Adriano de la SECA, «Colegio de jesuitas de Orense», Boletín de la Comisión Provincial de Monumentos Históricos y Artísticos (B.C.M.OR), tomo IX, núm. 190 y ss., Ourense, 1930-1932.

6 Véanse GRUPO NONO ART, Los gallegos y el Nuevo Mundo en la época virreinal, Barcelona, Nono-Art, s.a.-Banco Pastor, 1987; Antonio EIRAS RoEL, «Galicia e América dende o Quinto Centenario», Revista da Comisión Galega do Quinto Centenario (R.C.G.Q.C), núm. 1, Santiago de Compostela, 1989, pp. 13-19; Ofelia REY CASTELAO, «Os aportes demográficos a América nos séculos XVI, XVII e XVIII», en Galicia e América. Cinco séculos de historia, Santiago de Compostela, Consellería de Relacións Institucionais e Portavoz do Goberno-Consello da Cultura Galega, 1992, pp. 44-47; Antonio EIRAS RoEl y Ofelia REY CASTELAO, Los gallegos y América, Madrid, Mapfre, s.a., 1992, pp. 13-213; Fausto DoPICO, «Aportes e efectos demográficos da emigración a América (1750-1930)», en Galicia e América. Cinco séculos de historia, Santiago de Compostela, Consellería de Relacións Institucionais e Portavoz do Goberno-Consello da Cultura Galega, 1992, pp. 61-64; José Luis ANTA FELEZ, «Emigración gallega a América, siglo XVI, 15601599», en Primeras Jornadas Presencia de España en América: aportación gallega, Madrid, Deimos, 1989, pp, 229-249.

7 Véanse, entre otros muchos trabajos al respecto, Antonio EIRAS ROEL (Coord.): Emigración española y portuguesa a América, Alicante, Instituto de Cultura «Juan Gil-Albert», 1991; La emigración española a ultramar, 1492-1914, Madrid, Tabapress; Aportaciones al estudio de la emigración gallega. Un enfoque comarcal, Santiago de Compostela, Secretaría Xeral para as Relacións coas Comunidades Galegas da Xunta de Galicia, 1992; EIRAS Roel y ReY CASTELAo [6], pp. 215 y ss.; Xosé Antonio LóPEZ TABOADA, Economía e población en Galicia, A Coruña, Ediciós do Rueiro, 1979; La población de Galicia, 1860-1991, A Coruña, Fundación Caixa Galicia, 1996; María Xosé RODRíGUEZ GALDO, Galicia, país de emigración. La emigración gallega a América hasta 1930, Colombres, Archivo de Indianos, 1993; O fluxo migratorio dos séculos XVIII ó XX, Santiago de Compostela, Secretaría Xeral de Relacións coas Comunidades Galegas da Xunta de Galicia, 1995; Carlos SiXIREI PAREDES, A emigración, Vigo, Galaxia, 1988; Ramón VILlares y Marcelino FERNÁNDEZ, Historia da emigración galega a América, Santiago de Compostela, Secretaría Xeral de Relacións coas Comunidades Galegas, 1996.

\section{R. I., $1999, \mathrm{n}^{\circ} 216$}


rrespondientes al siglo XVII, de los cuales no hemos podido determinar o esclarecer su supuesta condición de indianos ni el origen de los capitales que dedicaron a la munificencia ${ }^{8}$. E incluso no desechamos la hipótesis de que existan donantes hasta ahora no identificados. Por consiguiente, aunque los datos anteriores resultan verosímiles, en consonancia con la discreta participación humana de Galicia en la empresa colonizadora, han de manejarse con la oportuna cautela que impone su provisionalidad.

Avanzando un poco más y deteniéndonos en la tercera columna del cuadro 1 comprobamos que de los once fundadores registrados, seis residían en distintas localidades del Virreinato de Perú y cuatro estaban asentados en el de Nueva España —el actual México-, distribución que guarda gran analogía con las opciones preferentes de destino de los gallegos que pasaron a Indias en los primeros siglos de la colonización ${ }^{9}$.

Atendiendo a su perfil profesional, el grupo presentaba la siguiente composición interna: cinco de los mecenas pertenecían al clero, siendo prelados cuatro de ellos; uno era escribano que actuaba en calidad de apoderado de un funcionario de la Administración Civil; otro ejercía como fiscal; había un rico propietario, y tres donantes de los que ignoramos su ocupación y status. La acusada prominencia de eclesiásticos en la nómina de fundadores resulta congruente con la notoria participación de los mismos en la experiencia colonizadora de este $\operatorname{siglo}^{10}$, que

\footnotetext{
8 Así ocurre con algunos de los que figuran en la «Relación...» que acompaña a la Estadística general de primera enseñanza correspondiente al decenio que terminó en 31 de diciembre de 1880, Madrid, Imprenta y Fundición de Manuel Tello, 1883 y en otros repertorios posteriores como: MINISTERIO DE GOBERNACIÓN. DIRECCIÓN GENERAL DE ADMINISTRACIÓN, Nuevos apuntes para el estudio y organización en España de las instituciones de beneficencia y previsión, Madrid, 1919; Estadística de la beneficencia particular de España correspondiente a los años 1922 al 1925 inclusive, Madrid, Establecimiento tipográfico Huelmes y $\mathrm{C}^{\mathrm{ia}}$, 1926; Estadística de la beneficencia particular de España correspondiente a los años 1926 al 1928 inclusive, Madrid, 1930; MINISTERIO DE EDUCACIÓN NACIONAL, Las fundaciones benéfico-docentes, Madrid, Comisaría General de Protección Escolar y Asistencia Social, 1959; ASOCIACIÓN DE DIRECTIVOS DE ENTIDADES NO LUCRATIVAS ESPAÑOLAS-CENTRO DE FUNDACIONES, Directorio de las Fundaciones españolas, Madrid, Ediciones Peninsular, 1986. Entre los donantes que dirigieron sus contribuciones hacia el sector educativo se encuentra Úrsula Menéndez de Tejada (Betanzos -A Coruña-, una escuela en 1629); Andrés Varela (Cervo -Lugo-, ayudas para huérfanos y estudiantes en 1641); Juan Rubinos Parga (Lourenzá -Lugo-, una escuela para niños en 1660); Mauro de Villarroel (Lourenzá -Lugo-, una escuela para niños en 1646); Antonio Pérez Castellanos (Quiroga -Lugo-, una escuela para niños en 1684); Juan García (Cerdedo -Pontevedra-, una escuela en 1612); Sebastián Parcero (Salceda de Caselas -Pontevedra-, una escuela para niños en 1650) y Bartolomé Barbeito ( Salvaterra -Pontevedra-, una escuela para niños en 1696).

9 Véanse, ANTA Felez [6], p. 232; Rey Castelao [6], p. 45; EIRAs Roel y Rey Castelao [6].

10 Véanse, Lino GÓMEZ CANEDO, Los gallegos en el gobierno, la milicia y la iglesia en América, Santiago de Compostela, Consellería de Relacións Institucionais e Portavoz do Goberno, 1991, especialmente pp. 88-136; Galicia y la evangelización de América [5]; GRUPO NONO ART [6], pp. 95-109; Isidro DUBERT GARCIA, «Galegos na Igrexa americana», en Galicia e América [4], pp. 72-75; EIRAs RoEl y Rey CASTElao [6], pp. 109-123.
} 
como es bien sabido adopta el sesgo de cruzada espiritual al servicio de la evangelización ${ }^{11}$.

En cuanto a su cronología, tomando como indicador el año de constitución o de formalización, las fundaciones y remesas se reparten de manera casi uniforme a lo largo de los cuatro cuartos del siglo, a razón de dos en el primero y tres en cada uno de los restantes. Sus fechas extremas de concreción se sitúan, respectivamente, en 1607 y en 1699 . Ahora bien, desde que adquieren carta de naturaleza muchas de ellas hasta que se materializa la donación transcurren habitualmente varios años, con relativa frecuencia décadas y aun en casos extremos el capital fundacional acaba extinguiéndose antes de hacerse efectiva la aportación, como consecuencia de interminables negociaciones y de enmarañados pleitos, cuando no de conductas mezquinas o de actitudes negligentes. Por imperativos formales no resulta pertinente que nos detengamos a analizar aquí la peripecia específica y diferencial de cada una de las fundaciones indianas, ni tan siquiera de aquellas más paradigmáticas. Pero a modo de síntesis, y asumiendo el riesgo que comporta toda generalización simplificadora acerca de un fenómeno tan variado y complejo, diremos que la tónica dominante se acomodó a un patrón dilatorio con amplios hiatos temporales entre la cesión de los bienes por parte de los donantes, su transferencia a Galicia, la recepción de los mismos por los beneficiarios y finalmente su inversión en la obra preconcebida, cuando esto último llegaba a producirse. Tales demoras obedecen a una casuística muy dispar, pero bajo la cual - por lo regular - subyace como denominador común la discordia entre los encargados de ejecutar las mandas fundacionales y sus destinatarios u otros agentes extraños, por mor de la divergencia de intereses particulares, que comúnmente acaban prevaleciendo frente a la voluntad de los propios benefactores ${ }^{12}$.

Respecto a la dimensión financiera de las fundaciones y legados, los datos disponibles no siempre poseen un grado óptimo de fiabilidad, muchas veces son imprecisos o fragmentarios e incluso en algún caso no corresponden a la fecha en

11 Véase el artículo ya clásico de Luisa CUESTA, «La emigración gallega a América», Arquivos do Seminario de Estudos Galegos, IV, Santiago de Compostela, 1932, particularmente las pp. 153-157.

12 Como apoyatura documental de estas afirmaciones y muestra ejemplificadora de lo expuesto véase lo ocurrido con las fundaciones de Domingo Araújo y Ferraz, Frai Diego de Hevia, Pedro Mondragón-Alonso de Santana y Mateo Segade. Para el primero: Jesús FERRo CoUSELO, «El convento de Santo Domingo de Orense», B.C.M.OR., tomo XIX, Ourense, 1957-58, especialmente pp. 203-215. Para el segundo: Vicente BELTRÁN DE HEREDIA, «Los benedictinos en la Universidad de Santiago». Boletín de la Real Academia Gallega (B.R.A.G.), núm. 181, A Coruña, 1 de marzo de 1926, pp. 12-19; Salvador CABEZA DE LEÓN y Enrique FERNÁNDEZ-VILLAMIL, Historia de la Universidad de Santiago de Compostela, Santiago de Compostela, C.S.I.C.-I.P.S.E.G., tomo I, vol. II, 1946 , p. 360 y tomo II, 1947 , pp. $139-145$ (existe reedición reciente). Para el tercero: RIVERA VÁZQUEZ [5], pp. 330-335 y DE LA SECA [5]. Y para el cuarto Armando CoTARELO VALLEDOR, «Lembranza biográfica de D. Mateo Segade Bugueiro», en Terra de Melide, Santiago de Compostela, Seminario de Estudos Galegos, 1933, especialmente pp. 639 y ss. (existe reedición reciente).

\section{R. I., $1999, \mathrm{n}^{\circ} 216$}


que se hizo efectiva la donación sino a otra muy posterior, cuando presumiblemente su capital inicial ya había decrecido o se había devaluado de manera ostensible. De cualquier modo, estos escollos no impiden apreciar la muy desigual contribución económica que realizan los distintos filántropos y, por consiguiente, discernir las diferencias patrimoniales que existen entre unas y otras fundaciones y la dispar magnitud de las remesas. Así, por ejemplo, mientras Mateo Segade Bugueiro cede todos sus cuantiosos bienes y los de su hermano para crear, dotar y sostener la Obra Pía de San Antonio de Melide ${ }^{13}$, y Domingo de Araújo Ferraz deja el remanente de sus posesiones - estimado en unos 130.000 ducados-para erigir un convento dominico en Ourense ${ }^{14}$, Benito Novoa remite al Colegio Fonseca de Compostela sólo 300 pesos ${ }^{15}$ y Pedro Martínez Rego lega 4.000 reales para establecer la Obra Pía de San Pedro de Arante ${ }^{16}$. Entre ambos extremos se sitúan los demás mecenas, algunos con una sola fundación o entrega y otros a su vez con varias (véase el cuadro 1 ).

Ahora bien, en materia económica, además de conocer el capital fundacional o la suma del legado, interesa saber si su importe se recibió o no en destino, y en el primer supuesto si llegó íntegro o ya mermado a sus beneficiarios. La información que suministran las fuentes manejadas para dilucidar estos aspectos es muy desigual, aunque lamentablemente, por lo regular, inconcreta, confusa o exigua. Sabemos, en efecto, que de las once fundaciones registradas llegaron partidas económicas a Galicia, pero sólo en una de ellas - que en realidad era un donativo- podemos asegurar que se recibió la dotación completa. En dos más, únicamente parte del monto adjudicado. Y en cuanto a las otras ocho carecemos de datos o estos no permiten esclarecer lo ocurrido. Ahondando un poco más en el tema, nos parece oportuno reparar en un caso al que quizá se asemejen algunos otros. Se trata de nuevo de la fundación promovida por Domingo Araújo Ferraz, que estaba dotada con un capital inicial estimado, como ya dijimos, en 130.000 ducados. Pues bien, según Ferro Couselo ${ }^{17}$, de tan copioso caudal monetario sólo llegaron a Ourense 4.000 ducados, y en opinión de Pardo Villar ${ }^{18}$, la partida cobrada no superó los 900 . Al margen de la disparidad entre las cantidades que sugieren estos dos autores, lo que en realidad sorprende es el enorme desajuste

13 Sobre esta fundación véanse: COTARELO VALLEDOR [12], en especial pp. 635-644; Eduardo Lence Santar y Guitián, La obra pía de San Antonio de Mellid, Mondoñedo, Centro de Acción Social Católica, 1928; Francisco TETTAMANCY, «Fundación de la obra-pía de San Antonio de la Villa de Mellid», B.R.A.G., nº 66, A Coruña, 1 de noviembre de 1912, pp. 144-149 y núm. 67 de 1 de diciembre de 1912, pp. 163-169.

14 Véase FERRo COUSELO [12].

15 Véase CABEZa de LeÓn y FernándeZ-Villamil [12]. 1849.

16 Archivo Diocesano de Mondoñedo, Obra Pía de San Pedro de Arante, libro núm. 1, 1688-

17 Ferro Couselo [12].

18 Aureliano Pardo Villar, «El convento de Santo Domingo de Orense», en B.C.M.OR., núm. 196, Ourense, enero-febrero de 1931, p. 162. 
que se advierte entre la suma donada y la recibida, cualquiera de las dos cifras que consideremos. Recortes y drenajes de esta naturaleza - aunque no tal vez de igual alcance- debieron experimentarlos también otras fundaciones coetáneas, a tenor de las vicisitudes por las que pasaron ${ }^{19}$, y que sin duda se vieron agravadas por la desaparición de los donantes, la desidia, cicatería y egoísmo de sus testamentarios, la ausencia de protección oficial y, por supuesto, el amplio espectro de maniobra financiera que posibilitaba la inmensidad del océano.

\section{DESTINATARIOS Y DESTINOS}

Si retomamos la información compendiada en el cuadro 1, comprobamos que como beneficiarios de las fundaciones y remesas educativas de los indianos gallegos en su fase auroral figuran en primer término los vecinos y parientes de los donantes, a continuación aparecen las congregaciones religiosas (jesuitas, dominicos, benedictinos y franciscanos), en tercer lugar se encuentra la Universidad y por último otros receptores no identificados, quizás pertenecientes al primer grupo. Este reparto de adjudicatarios en orden de prelación decreciente ofrece claves para dilucidar los vínculos de enlace que mantenían engarzados a los indianos desde la distancia con las instituciones y las gentes de sus lugares de procedencia, cuestión ésta en la que abundaremos en el próximo epígrafe.

Los destinos concretos que les confieren a sus donaciones se distribuyen del siguiente modo: dotación de quince becas para contribuir a sufragar estudios superiores, creación de dos colegios religiosos, fundación de dos cátedras universitarias, erección de dos conventos, establecimiento de tres obras pías para la enseñanza de las primeras letras - una de ellas provista además de siete lectorías para la formación de clérigos-, una escuela elemental y tres partidas monetarias de las que dos se le transfieren al Colegio Fonseca de Santiago de Compostela y la tercera a la localidad de Iria Flavia para «colación» de los niños de su escuela y gratificación del maestro.

En el plano institucional, la enseñanza eclesiástica regular y la universitaria emergen como principales focos de atracción de la munificencia indiana en el área educacional durante el siglo XVII. Este comportamiento, propio de la mentalidad hegemónica de la época, resulta además razonable si tenemos en cuenta las estrechas relaciones que existían entre los donantes y las instituciones favorecidas. Piénsese, por ejemplo, que cuatro benefactores (Frai Diego de Hevia, Alonso de la Peña, Mateo Segade y Benito Novoa) estaban ligados por formación

19 Véanse al respecto: Cotarelo VAlledor [12], pp. 639-644; Rivera VÁZquez [5], pp. 330335 y 357-367; DE LA SECA [5]; y Enrique FERNÁNDEZ-VILLAMIL, «Noticias sobre la fundación y desarrollo del Colegio de la Compañía de Jesús en Pontevedra», El Museo de Pontevedra, XXXV, Pontevedra, 1981, pp. 364-406.

\section{R. I., $1999, \mathrm{n}^{\circ} 216$}


y/o magisterio a la Universidad de Santiago de Compostela, cuna y cantera desde fecha temprana de clérigos y funcionarios civiles que pasaron a Indias ${ }^{20}$. Tres de los anteriormente citados destinaron su donativo a la propia casa de estudios universitarios a la que habían pertenecido y el cuarto lo hizo a su localidad de procedencia, fundando una Obra Pía y protegiendo al Convento donde probablemente había cursado su primera enseñanza ${ }^{21}$. Ya escribimos más arriba que, a su vez, cinco de los mecenas eran eclesiásticos, y podemos añadir ahora que al menos otros dos (Domingo de Araújo y Alonso de Santana) mantenían, respectivamente, lazos de amistad y parentesco con miembros de las congregaciones religiosas a las que otorgaron sus legados 22 .

De este modo se explica la dirección específica y preferente que los primeros indianos les imprimieron a sus donaciones en la esfera educativa, en contraste con la manera de proceder de sus continuadores siglos después, por razones que ya hemos tenido oportunidad de explicitar en otro lugar ${ }^{23}$.

\section{MOTIVACIONES Y ANHELOS}

Los móviles que impulsaron a los indianos a crear fundaciones docentes y a vehicular sus aportaciones hacia el área educativa no parece que difiriesen, en lo sustancial, de los agentes que operaron como factores de estímulo en otros donantes no desplazados a Indias. En ambos podemos apreciar, de forma velada o expresa, la concurrencia de una serie de motivaciones de índole general, más o menos comunes y permanentes, asociadas a otras de carácter particular y diferencial que varían en razón de los individuos y mudan con los tiempos.

Centrándonos en el primer grupo, podemos afirmar que los distintos ben̉efactores evidencian con su conducta - si bien no siempre lo acreditan por escrito de manera explícita- ser partícipes de una concepción valorativa de la educación de signo positivo. Y es precisamente esa estimación compartida la que genera y alimenta en ellos el propósito de contribuir al desarrollo y la promoción de la enseñanza, propiciando que su mecenazgo se polarice en dirección hacia el ámbito académico.

También de un modo generalizado, aunque con gradientes y matices, el grueso de los donantes patentiza una virtual proclividad filantrópica y altruista al

20 Como en su día hizo notar Luisa CuESTA, ya en 1604 el VII Conde de Lemos -Presidente del Consejo de Indias- escribió a la Universidad de Santiago de Compostela socilitándole personas aptas para las audiencias de Indias. CUESTA [11], pp. 154 y 194. Para un tratamiento más amplio y actualizado del tema véase GómEZ CANEDO [10], pp. 93 y passim; EIRAS ROEL y REY CASTELAO [6], pp. 109 y ss.

21 Véase Cotarelo Valledor [12], pp. 584 y 636.

22 Véanse Ferro Couselo [12], pp. 206-208 y Rivera VÁZquez [5], p. 131.

23 PeÑa SaAVEDRa [1], vol. I, pp. 303-351. 
efectuar sus aportes ${ }^{24}$. No obstante, la munificencia que inspira su proceder tiene por lo regular una proyección más localista o tribal que propiamente ecuménica $o$ universalista, como permite inferir una revisión, por superficial que sea, del elenco de beneficiarios a los que se orienta con exclusividad o primacía. En ocasiones el círculo queda acotado prioritariamente a los parientes próximos ${ }^{25}$. Otras veces la acción se hace extensiva al colectivo vecinal ${ }^{26} \mathrm{y}$, de ordinario, presenta además ribetes de obra caritativa a favor de los más humildes ${ }^{27}$. En un tercer supuesto la destinataria es alguna de las instituciones a las que perteneció el indiano en su momento $^{28} \mathrm{o}$ a las que aún permanece adscrito ${ }^{29}$. Y no faltan casos en los que prima el criterio de la amistad, la simpatía, la devoción o la identidad de creencias para la asignación y el disfrute del donativo ${ }^{30}$. En cualquiera de las situaciones mencionadas, el donante comparte su fortuna con los más allegados, y lo hace imbuído por sentimientos filantrópicos y caritativos o por otras causas motivas, de naturaleza no tan altruista, que a continuación vamos a exponer dentro ya del segundo grupo al que antes nos referimos.

Si revisamos las escrituras de fundación localizadas, correspondientes a los indianos gallegos del siglo XVII, constatamos que no pocos presuntos filántropos al efectuar su legado aspiraban a rentabilizarlo espiritualmente ${ }^{31}$, tratando de

24 Uno de los ejemplos más genuinos de «pura» filantropía y de magnánimo desprendimiento es el que ofrece Jorge de Andrade. Véase FERNÁNDEZ-VILLAMIL [19], en particular p. 375.

25 Véanse los casos de Mateo Segade, Alonso de la Peña y Domingo de Araújo, respectivamente en TetTamancy[13], p. 149; Manuel Bandín Hermo, El Obispo de Quito Don Alonso de la Peña Montenegro (1596-1687). Madrid, C.S.I.C., 1951, pp. 461-462; y FERRO COUSELO [12], p. 205.

26 Jorge de Andrade, por ejemplo, manifiesta su intención de fundar el Colegio de la Compañía de Jesús en la villa de Pontevedra, de donde es natural, «para mexor educación de los naturales della». Véase escritura fundacional en FERNÁNDEZ-VILLAMIL [19], p. 373. Cfr. asimismo, para Alonso de Santana, DE LA SECA [5], entrega publicada en el núm. 192 de mayo-junio de 1930, p. 70, y los casos de Pedro Martínez Rego, Antonio Ramos de Soto y Andrés Otero en el cuadro 1.

27 Véase como muestra la escritura fundacional de Mateo Segade en TETTAMANCY [13], pp. 146 y 149.

28 Véanse los ejemplos de Benito Novoa y Salgado y Alonso de la Peña en CABEZA DE LEÓN y FERNÁNDEZ-VILLAMIL [12], t. I, vol. II, pp. 36-43; y BANDín HERMo [25], pp. 20 y ss. y 456.

29 Es el caso del monje benedictino Frai Diego de Hevia que crea una cátedra en la Universidad compostelana para ser ocupada por los frailes de su misma Orden radicados en el convento de San Martín Pinario. Véase BELTRÁN DE HEREDIA [12], pp. 12-16.

30 Así ocurrió con las fundaciones de Jorge de Andrade, Alonso de Santana o Domingo de Araújo. Véanse, para la primera: FernÁNDEZ-VIllamil [19], pp. 365-366 y 381, y RIVERA VÁZQuEZ [5], p. 358. Para la segunda: DE LA SECA [5], pp. 11 y ss. y RIVERA VÁZQUEZ [5], p. 358. Y para la tercera FERRO COUSELO [12], pp. 206-208.

31 De uno o de otro modo coincidieron en poner de relieve la preeminencia de esta motivación espiritual en los donantes de los siglos XVI y XVII, como también del medievo, varios autores. Entre ellos cabe citar a Dámaso LARIO, «Mecenazgo de los Colegios Mayores en la formación de la burocracia española (siglos XIV-XVIII)», Universidades españolas y americanas. Época colonial, Valencia, Generalitat Valenciana-C.S.I.C., 1987, pp. 277-309; Antonio VIÑAO FRAGO, «Filantropía y educación. Fundaciones docentes y enseñanza elemental (siglos XVIII-XIX)», en L'enseignement 
obtener como contrapartida la salvación para sí mismos ${ }^{32}$ y acaso también para sus familiares ${ }^{33}$. Mas, si por alguna contingencia no fuese posible colmar semejante anhelo con el acto e importe de la donación, confiaban al menos en que su óbolo resultase suficiente para eludir la irreversible condena eterna, y con los réditos que produjese en forma de misas, plegarias y rezos asegurar la rápida redención de sus almas ${ }^{34}$, las de sus parientes y demás obligaciones ${ }^{35}$. Claro que la filantropía y la caridad afloraban de nuevo aquí, al expresar los donantes su voluntad de hacer extensivas a todas las ánimas del Purgatorio las gracias derivadas de los sufragios y las oraciones con que gravaban a sus beneficiarios ${ }^{36}$.

Pero no era sólo la gloria celestial y la previa reparación de culpas para su logro el único fin espiritual que perseguían los indianos al establecer sus fundaciones. Había algunos cuyo principal interés radicaba en difundir la fe en su localidad de procedencia, con objetivos proselitistas solapados o explícitos ${ }^{37}$. Otros pretendían favorecer la implantación de una Orden religiosa en un territorio ${ }^{38}$, afianzar su presencia en una zona ${ }^{39} \mathrm{o}$ incluso reforzar su peso relativo en el seno de una institución ${ }^{40}$. No faltaron los que se proponían facilitar estudios eclesiásticos a parientes o vecinos ${ }^{41}$, sin duda para procurar su promoción social y consolidar la acción apostólica. Y casi todos querían en primera y última instancia cooperar con su obra a la «mayor gloria y honra de Nuestro Señor» ${ }^{42}$ y por extensión de sus santos ${ }^{43}$.

Desde una óptica más laica y profana, cualquier fundación representaba una fórmula peculiar y exitosa de retorno a la patria de los ancestros, sustitutoria o

\footnotetext{
primaire en Espagne et en Amerique Latine du XVIII siècle a nos jours-Politiques èducatives et Rèalités scolaires-, Tours, Publications de L'Université de Tours, 1986, pp. 65-79, y Domingo L. GONZÁLEZ LOPO, «Os froitos da emigración: as fundacións filantrópicas dos indianos galegos», en Galicia e América. Cinco séculos de historia, Santiago de Compostela, Consellería de Relacións Institucionais e Portavoz do Goberno-Consello da Cultura Galega, 1992, pp. 213-216.

32 Véase DE LA SECA [5], pp. 11 y ss.

33 Véase escritura fundacional de Alonso de la Peña en BANDÍn HERMo [25], p. 455.

34 BANDÍN HERMO [25], p. 456.

35 TeTTAMANCY [13], p. 167, y DE LA SECA [5], p. 69.

36 Véanse FernándeZ-Villamil [19], p. 375; TetTamancy [13], p. 147, y Bandín Hermo [25], pp. 455 y 462.

37 Véase la carta de Jorge de Andrade desde Lima, datada el 31 de mayo de 1640, en FernándeZ-VILlamil [19], p. 381. También Bandín HeRmo [25], p. 461 y DE LA SECA [5], p. 144.

38 Son los casos de Domingo de Araújo o de Alonso de Santana.

39 Véase escritura de fundación de Mateo Segade, en TETTAMANCY [13], p. 146.

40 Por ejemplo en la fundación de frai Diego de Hevia. Véase nota 29.

41 Véase TetTamancy [13].

42 Véanse FernándeZ-Villamil [19], p. 381; Bandín HeRmo [25], p. 454, y DE LA SECA [5], p. 37.

43 Véase BANDín HERMo [25], p. 462.
} 
complementaria del regreso físico ${ }^{44}$. Con ella, además de la gloria del paraíso - tan codiciada como incierta-, los indianos podían conseguir la nada desdeñable inmortalidad mundana, perpetuándose en la memoria de sus coparroquianos $\mathrm{y}$, con algo de suerte, también de otras gentes ${ }^{45}$ en calidad de modélicos protectores, para lo cual no vacilaban en idear eficaces estrategias ${ }^{46}$. Incluso, si había lugar a ello, cabía la posibilidad de poder saldar deudas de gratitud ${ }^{47}$ o cumplir compromisos contraídos antes de la partida. Y cómo no, descargar la conciencia de culpabilidades por presuntas faltas en las que hubiesen incurrido ${ }^{48}$.

Quizá existieron otras razones ocultas, confesables o no, que probablemente nunca conoceremos por el silencio de los donantes o por la carencia de fuentes ajenas a ellos. Pero, en todo caso, como condición previa tuvo que ocurrir un hecho que de no llegar a producirse restaría validez explicativa a cualquiera de las motivaciones aquí invocadas. Nos referimos al mantenimiento de los lazos de unión de los ausentes con sus respectivas localidades de procedencia o, si se prefiere, a la no consumación de su desarraigo definitivo ${ }^{49}$, por diversas causas que ahora resultaría demasiado prolijo inventariar. Esto posibilitó la transferencia de remesas materiales e inmateriales hacia su tierra y la creación, ya en fecha temprana, de fundaciones de carácter educativo, como variante y destino de tales remesas ${ }^{50}$, por cierto casi siempre teñidas de un inequívoco y desaforado afán de

44 Escribía Luisa Cuesta respecto a los donantes que realizaron sus dádivas a la Universidad compostelana: «del mismo modo que desean que sus cenizas reposen en el país natal donde duermen el sueño eterno sus antepasados, ansían que su memoria viva entre sus compañeros y profesores, unido a la gratitud por sus donativos y fundaciones». CUESTA [11], p. 154.

45 Eduardo Álvarez Carballido señala que era propósito de Mateo Segade «dejar en su patria un monumento que a la vez que perpetuase su memoria fuese útil a sus paisanos, y asegurase a la posteridad una instrucción sólida y gratuita». Eduardo ÁlvaREZ CARBALLIDO, «Gallegos ilustres. El Arzobispo de Méjico D. Mateo Segade y Bugueiro», en Galicia Diplomática, tomo III, Santiago de Compostela, 29 de abril de 1888, p. 132.

46 Por ejemplo, mandando esculpir su nombre en lápida recordatoria o instituyendo una misa de aniversario. Véanse los casos de Pedro Mondragón-Alonso de Santana y Alonso de la Peña, en DE LA SeCA [5], p. 68; GRUPo Nono ART [6], pp. 64-65; BANDín HeRmo [25], pp. 298, 357-358 y 456, y Antonio NeIRA DE MosQuera, Monografías de Santiago y dispersos temas compostelanos (1844-1852), Santiago de Compostela, Bibliófilos Gallegos, 1950, p. 230, nota 17.

47 Véase RIVERA VÁzQUEZ [5], p. 331.

48 Véase DE LA SECA [5], p. 14.

49 Los autores que se ocuparon de estudiar las fundaciones de los indianos gallegos aluden, en relación a este punto, al recuerdo y a la añoranza de la aldea o de la patria de procedencia y a su amor hacia ellas. Véanse Rivera VÁZQUEZ [5], p. 359; FerNÁNDEZ-VILlamil [19], p. 380, y GONZÁLEZ LOPO [31], p. 213.

50 Hubo también indianos gallegos que, tal vez, más apegados a la tierra de destino y comprometidos con ella ejercieron allí su filantropía docente. En el siglo XVII cabe citar, entre otros, al arzobispo de México Francisco Aguiar y Seijas o al obispo de Santiago de Cuba Diego Evelino de Compostela. Véanse sobre ellos: Francisco A. RUBio DURÁn y Braulio L. Flores MORÓn, «Un gallego en el arzobispado de México: Francisco de Aguiar y Seixas», en Primeras Jornadas Presencia de España en América: aportación gallega, Madrid, Deimos, p. 185; Benigno TeIJEIRO 
progreso. En realidad, lo que comenzaba a tomar cuerpo en el siglo XVII era sólo el preludio de una acción de muy superior alcance y de insospechado relieve que iría dilatándose gradualmente en el transcurso de las décadas para frisar su cénit en el primer tercio de la presente centuria.

In this article we make a first recount and analysis of the philantropic actions undertaken in the educational area by indiano galicians during the $17^{\text {th }}$ century, prelude of a long duration process that will go on until our days. The sources used have a documentary and bibliographic character. The content of the work is focused on the personal and professional profile of donnors, their geographic settlement, the chronologic distribution of the contributions they made, their financial dimension, the payees and specific uses that they confered them, the realizations that they started and the motivations underlying their remittances. The provisional balance shows a total of 12 benefactors and wealth relatively substantial and plural.

MARTÍNEZ, Gallegos ilustres en América desde la conquista hasta nuestros días. Notas biográficas. Buenos Aires, Tip. de Antonio Cursach, 1901, p. 20; GRUPO NONO ART [6], pp. 73-74, y GómEZ CANEDO [10], p. 93.

R. I., $1999, \mathrm{n}^{\circ} 216$ 


\begin{tabular}{|c|c|c|c|c|c|c|c|}
\hline \multirow{9}{*}{ 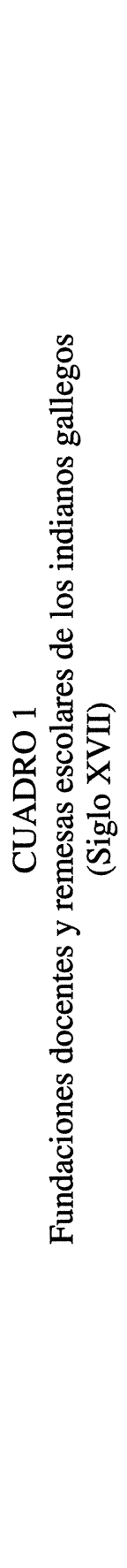 } & 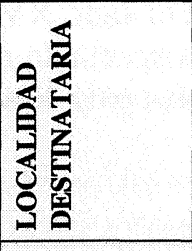 & 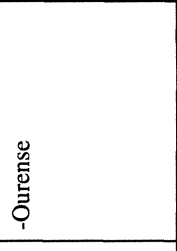 & 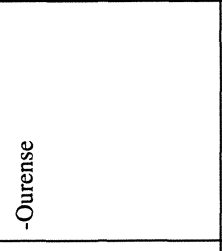 & 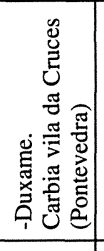 & & 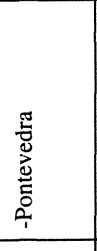 & 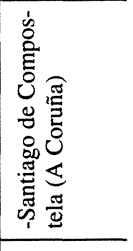 \\
\hline & 起 & 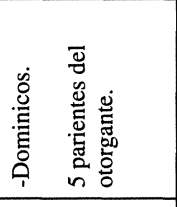 & 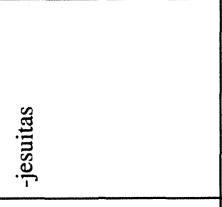 & 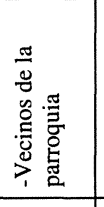 & 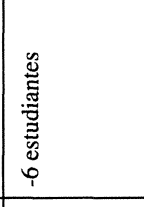 & 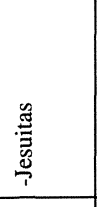 & 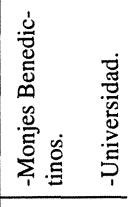 \\
\hline & 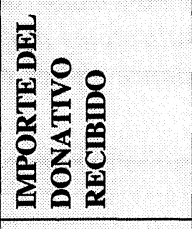 & 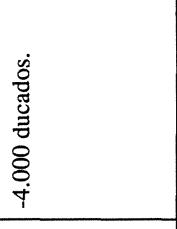 & 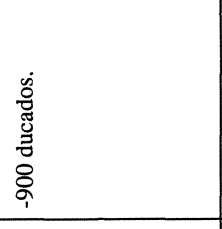 & & & 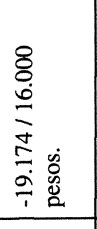 & \\
\hline & 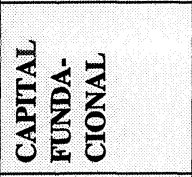 & 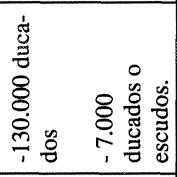 & 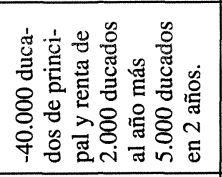 & & & 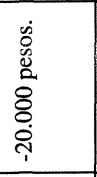 & 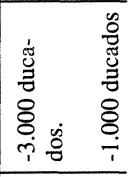 \\
\hline & 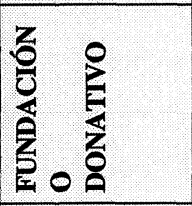 & 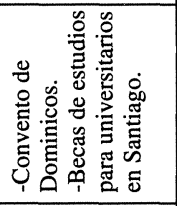 & 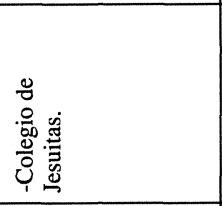 & 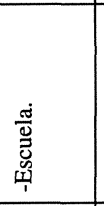 & 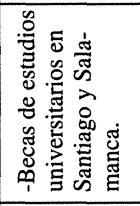 & 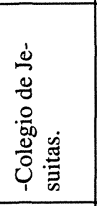 & 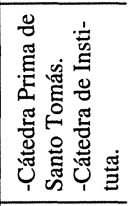 \\
\hline & 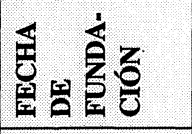 & ప్రీ & $\underline{\widetilde{\Xi}}$ & ఫ్రి & $\stackrel{8}{.}$ & 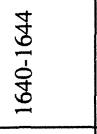 & 号 \\
\hline & 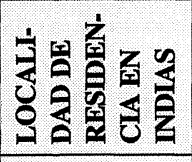 & 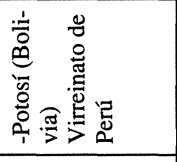 & 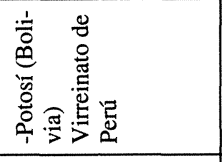 & 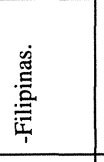 & 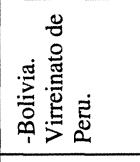 & 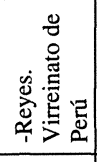 & 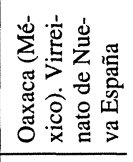 \\
\hline & 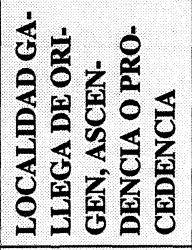 & 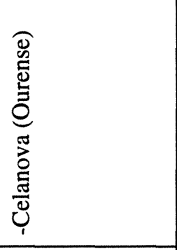 & 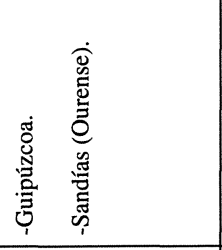 & 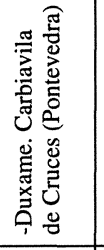 & 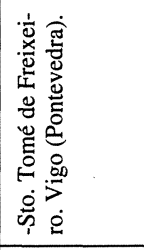 & 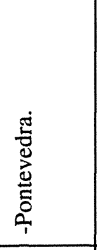 & 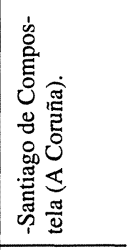 \\
\hline & 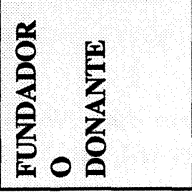 & 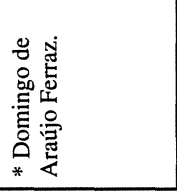 & 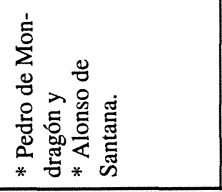 & 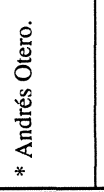 & 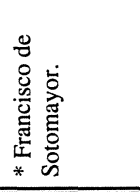 & 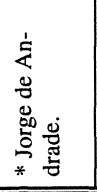 & 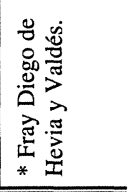 \\
\hline
\end{tabular}

R. I., $1999, \mathrm{n}^{\circ} 216$ 


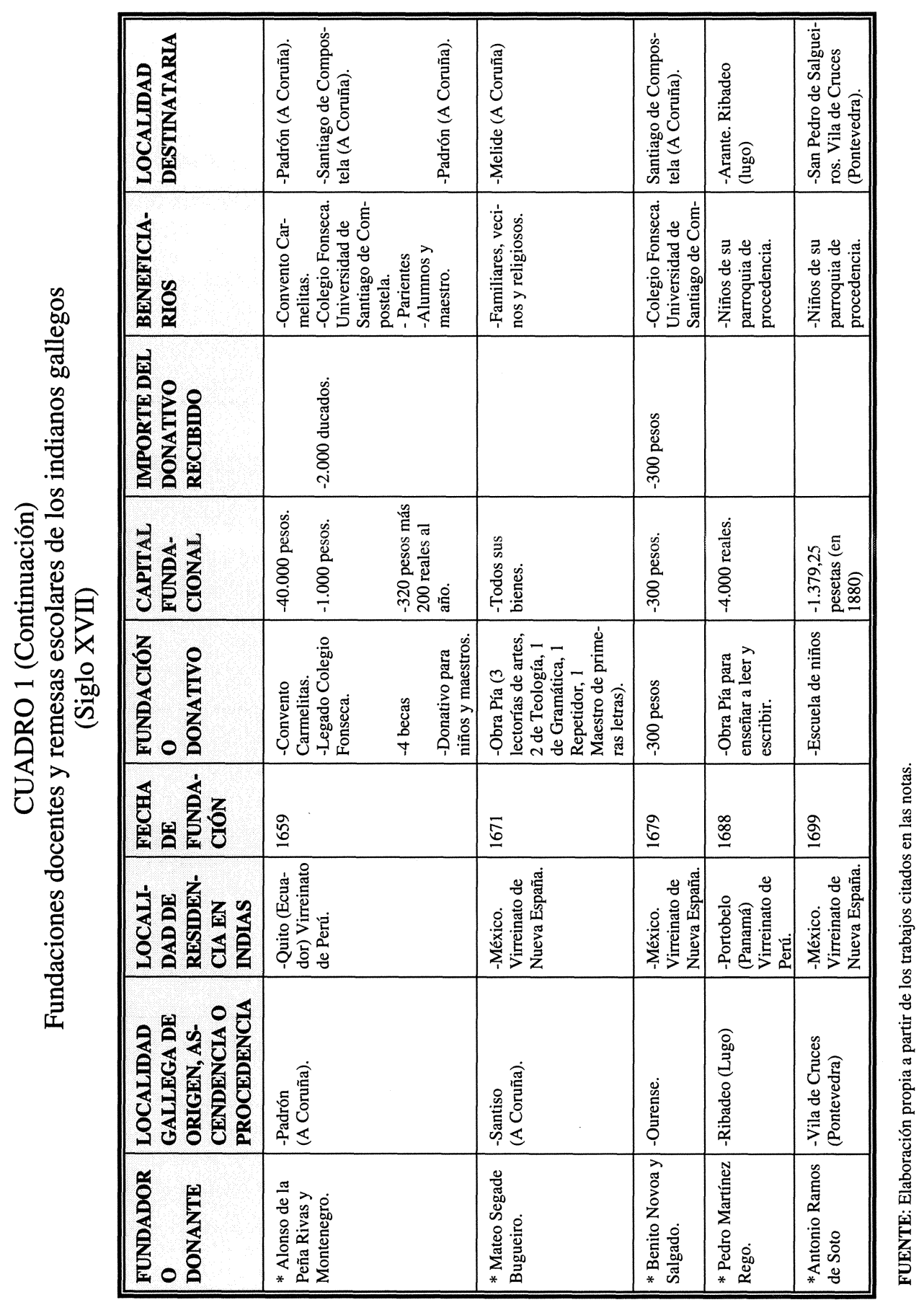

R. I., 1999, n. 216 